\title{
Prevention of Lipotoxicity in Pancreatic Islets with Gammahydroxybutyrate
}

Justin Hou Ming Yung ${ }^{1}$ (D) Lucy Shu Nga Yeung ${ }^{1}$, Aleksandar Ivovic ${ }^{1}$, Yao Fang Tan ${ }^{1}$, Emelien Mariella Jentz ${ }^{2}$, Battsetseg Batchuluun 1, Himaben Gohil ${ }^{1}$, Michael B. Wheeler ${ }^{1}$, Jamie W. Joseph ${ }^{2} \mathbb{D}$, Adria Giacca 1,3,4,5,*,t and Mortimer Mamelak $6, *,+$

1 Department of Physiology, University of Toronto, Toronto, ON M5S1A8, Canada; justin.yung@mail.utoronto.ca (J.H.M.Y.); lucy.yeung@mail.utoronto.ca (L.S.N.Y.); a.ivovic@mail.utoronto.ca (A.I.); yao.tan@utoronto.ca (Y.F.T.); battsetseg.batchuluun@gmail.com (B.B.); hima.gohil@mail.utoronto.ca (H.G.); michael.wheeler@utoronto.ca (M.B.W.)

2 School of Pharmacy, University of Waterloo, Kitchener, ON N2G1C5, Canada; emjentz@uwaterloo.ca (E.M.J.); jamie.joseph@uwaterloo.ca (J.W.J.)

3 Department of Medicine, University of Toronto, Toronto, ON M5S1A8, Canada

4 Institute of Medical Science, University of Toronto, Toronto, ON M5S1A8, Canada

5 Banting and Best Diabetes Centre, University of Toronto, Toronto General Hospital, Toronto, ON M5S12C4, Canada

6 Department of Psychiatry, University of Toronto, Toronto, ON M6A2E1, Canada

* Correspondence: adria.giacca@utoronto.ca (A.G.); m.mamelak@utoronto.ca (M.M.); Tel.: +1-(416)-978-0167 (A.G.)

+ These authors contributed equally to this paper.

check for updates

Citation: Yung, J.H.M.; Yeung, L.S.N.; Ivovic, A.; Tan, Y.F.; Jentz, E.M.; Batchuluun, B.; Gohil, H.; Wheeler, M.B.; Joseph, J.W.; Giacca, A.; et al. Prevention of Lipotoxicity in Pancreatic Islets with Gammahydroxybutyrate. Cells 2022, 11, 545. https://doi.org/10.3390/ cells11030545

Academic Editor:

Victoriano Baladrón

Received: 16 December 2021

Accepted: 30 January 2022

Published: 4 February 2022

Publisher's Note: MDPI stays neutral with regard to jurisdictional claims in published maps and institutional affiliations.

Copyright: (c) 2022 by the authors. Licensee MDPI, Basel, Switzerland. This article is an open access article distributed under the terms and conditions of the Creative Commons Attribution (CC BY) license (https:// creativecommons.org/licenses/by/ $4.0 /)$.

\begin{abstract}
Oxidative stress caused by the exposure of pancreatic ß-cells to high levels of fatty acids impairs insulin secretion. This lipotoxicity is thought to play an important role in $B$-cell failure in type 2 diabetes and can be prevented by antioxidants. Gamma-hydroxybutyrate (GHB), an endogenous antioxidant and energy source, has previously been shown to protect mice from streptozotocin and alloxan-induced diabetes; both compounds are generators of oxidative stress and yield models of type-1 diabetes. We sought to determine whether GHB could protect mouse islets from lipotoxicity caused by palmitate, a model relevant to type 2 diabetes. We found that GHB prevented the generation of palmitate-induced reactive oxygen species and the associated lipotoxic inhibition of glucose-stimulated insulin secretion while increasing the NADPH/NADP+ ratio. GHB may owe its antioxidant and insulin secretory effects to the formation of NADPH.
\end{abstract}

Keywords: oxidative stress; ß-cell; lipotoxicity; gamma-hydroxybutyrate (GHB); type 2 diabetes; NADPH

\section{Introduction}

Type 2 diabetes is characterized by insulin resistance and defective insulin secretion. Obesity is the major predisposing factor to type 2 diabetes and accounts for the excessive release of fatty acids from the expanded adipose tissue mass. High plasma levels of nonesterified fatty acids (NEFA) are known to cause insulin resistance, but the prolonged exposure of pancreatic ß-cells to high fatty acid levels also impairs the insulin-producing capacity of these cells by a process that likely involves the generation of oxidative stress [1] B-cell damage by fatty acids and the subsequent reduction in insulin secretion appears to be an early event in the pathogenesis of hyperglycemia in type 2 diabetes. There is now good evidence that antioxidants can prevent the development of oxidative stress in B-cells in response to fatty acids and that this can maintain the insulin-producing capacity of these cells [1,2]. Previous work in our laboratory has demonstrated that lipotoxicity is caused by the activation of islet NADPH oxidase by long-chain fatty acids and the ensuing formation of the superoxide radical and aldehydes. Antioxidants such as $n$-acetylcysteine, taurine, and tempol were shown to prevent the toxic effects of this oxidative stress $[1,2]$. 
We now set out to determine whether gamma-hydroxybutyrate (GHB), an intermediary metabolite in all living cells, can also prevent the damage to pancreatic islet cells, which occurs naturally in diabetes in response to high levels of fatty acids [3,4]. Previous studies have revealed that GHB can prevent the development of diabetes in mice challenged by streptozotocin and alloxan, two toxic agents that are employed to develop models of type 1 diabetes and that are known to generate oxidative stress in pancreatic islets $[5,6]$. Based on its capacity to prevent tissue damage following exposure to ionizing radiation or high oxygen pressure, Laborit [7] proposed that GHB owed its antioxidant properties to the activation of the pentose phosphate shunt (PPS) and the formation of the reducing coenzyme NADPH. Taberner et al. [8] confirmed that this was, in fact, the case, and GHB's antioxidant powers have been repeatedly demonstrated in the brain and other tissues, but it has never been clear just how GHB activates the shunt [3,4].

In this study, GHB's protective actions were examined in an in vitro model of $B$-cell lipotoxicity, which, as stated above, is relevant to type 2 diabetes. GHB was shown to block the formation of reactive oxygen species (ROS) and to prevent the reduction in glucose-stimulated insulin secretion (GSIS) produced by palmitate, the most common saturated NEFA.

GHB is on the market under the trade name $X_{y r e m}{ }^{\circledR}$ and has been used safely every night for many years by patients with narcolepsy [9]. GHB's capacity to protect the insulinproducing ß-cells of the pancreas from the toxic effects of high plasma levels of fatty acids may open the door to further investigation of this or related compounds as adjuvants in the treatment of type 2 diabetes.

\section{Materials and Methods}

\subsection{Animals}

All procedures were approved by the Animal Care Committee of the University of Toronto (animal protocol number: 20011526, approved 30 March 2021). Islets of 11-13 weeks old male C57BL/ 6 mice (Jackson Laboratory 000664) were isolated via collagenase (Sigma Aldrich Burlington, MA, USA, Cat. \#C9263) injection into the common bile duct as previously described [2,10-12].

All mice were co-housed in cages containing 2-4 mice, and had access to water and standard chow (Envigo Teklad Global 18\% Protein Rodent Diet (2918), Madison, WI, USA) ad libitum. Mice were not fasted prior to euthanasia and sample collection. Euthanasia was achieved by an overdose $(0.2 \mathrm{~mL} /$ mouse) of ketamine:xylazine:acepromazine (150 mg/kg:5 mg/kg:1 mg/kg) cocktail injected intraperitoneally in isofluorane anesthetized mice. Islet isolation then commenced.

\subsection{Islet Treatment In Vitro}

After 1 hr recovery in RPMI1640 (without antioxidants) with 7\% fetal bovine serum (FBS) containing $10 \mathrm{mM}$ HEPES, freshly isolated islets from C57BL/6 mice were incubated for $48 \mathrm{hrs}$ at $37{ }^{\circ} \mathrm{C}$ and $5 \% \mathrm{CO}_{2}$ with: (1) control islet medium; (2) palmitate $(0.4 \mathrm{mM})$; (3) palmitate $(0.4 \mathrm{mM})$ and GHB $(5 \mathrm{mM})$ or (4) control and GHB (5 mM). 48 hrs exposure of islets to this concentration of palmitate results in impairment in insulin secretion in islets [1]. The concentration of GHB was determined based on previous literature $[3,8]$. Health Canada exemption number for GHB: 51650.03.21.

\subsection{Glucose-Stimulated Insulin Secretion (GSIS) Assay}

Following islet treatment in vitro, islets were incubated in Krebs Ringer buffer containing $10 \mathrm{mM}$ HEPES (KRBH) with $2.8 \mathrm{mM}$ glucose for $1 \mathrm{hr}$ for recovery. GSIS was then evaluated by incubation of islets in $\mathrm{KRBH}$ containing: $6.5 \mathrm{mM}$ (basal glucose concentration for mice) or $22 \mathrm{mM}$ glucose (maximum stimulatory concentration) in duplicate for $2 \mathrm{hrs}$. Supernatant insulin was measured with Linco's RIA kit (EMB Millipore, Billercia, MA, USA, Cat. \#RI-13K). 


\subsection{Reactive Oxygen Species (ROS) Measurement}

ROS were detected with dihydro-dichlorofluorescein diacetate (H2DCF-DA) (Invitrogen, Waltham, MA, USA, Cat. \#C6827). Following islet treatment in vitro, islets were incubated with $10 \mu \mathrm{M}$ of H2DCF-DA in $2.8 \mathrm{mM}$ glucose in KRBH for $20 \mathrm{~min}$. After washing with $\mathrm{KRBH}$, fluorescence was measured at $490 \mathrm{~nm}$ excitation and $510 \mathrm{~nm}$ emission. Ten islets were measured per treatment.

\subsection{Oxygen Consumption Rate}

Oxygen consumption rate was measured with Seahorse XF cell Mito Stress Test (Seahorse Bioscience, Billerica, MA, USA) according to manufacturer's instructions. Prior to analysis, islets were preincubated in $525 \mu \mathrm{L}$ of XF Base Medium (Seahorse Bioscience) containing $2 \mathrm{mM}$ glutamine, $1 \mathrm{mM}$ sodium pyruvate and $16.5 \mathrm{mM}$ glucose at $37^{\circ} \mathrm{C}$ without $\mathrm{CO}_{2}$. Four separate compounds were then injected sequentially: (1) glucose (final concentration $22 \mathrm{mM}$ ); (2) oligomycin, an ATP synthase inhibitor, (final concentration $5 \mu \mathrm{M}$ ); (3) FCCP, an uncoupling agent, (final concentration $2 \mu \mathrm{M}$ ); and (4) antimycin (final concentration $5 \mu \mathrm{M}$ ) with rotenone (final concentration $5 \mu \mathrm{M}$ ), a cellular respiration and electron transport chain inhibitor. Five OCR measurements were taken after each injection.

\subsection{NADPH and NADP+ Measurement}

Islet NADPH and NADP+ levels were measured with Sigma Aldrich NADP+/NADPH Assay Kit (Sigma Aldrich, Burlington, MA, USA, Cat. \#MAK312) according to the manufacturer's instructions. Prior to analysis, islets were collected and washed twice in $2.8 \mathrm{mM}$ glucose in $\mathrm{KRBH}$. After washing with $\mathrm{KRBH}$, islets were frozen using a dry ice-ethanol bath for at least $5 \mathrm{~min}$. Samples were then stored at $-80{ }^{\circ} \mathrm{C}$ until analysis.

\subsection{Statistical Analysis}

Data are presented as means \pm SE. Shapiro-Wilk tests on Prism 9 (La Jolla, CA, USA) were run first to determine normality of data. Nonparametric or parametric analyses were performed accordingly. One-way nonparametric analysis of variance (ANOVA on ranks) or parametric ANOVA for repeated measurements (when appropriate) followed by Tukey's test was used to compare differences between treatments. Calculations were performed using SAS (Cary, NC, USA).

\section{Results}

Figure 1 illustrates the protective effect of $5 \mathrm{mM}$ GHB on insulin secretion in this in vitro model. Palmitate significantly decreased GSIS as expected. Despite exposure to palmitate, islets concomitantly treated with GHB maintained normal levels of insulin secretion. GHB alone did not affect insulin secretion.

Figure 2a,b illustrate the significant increase in ROS generated in mouse islets treated with palmitate and the absence of this effect in islets concomitantly treated with GHB.

Figure 3 reveals that palmitate tended to reduce islet oxygen consumption and that this appeared to be prevented by GHB. GHB alone also tended to decrease oxygen consumption. However, none of these effects were statistically significant. 


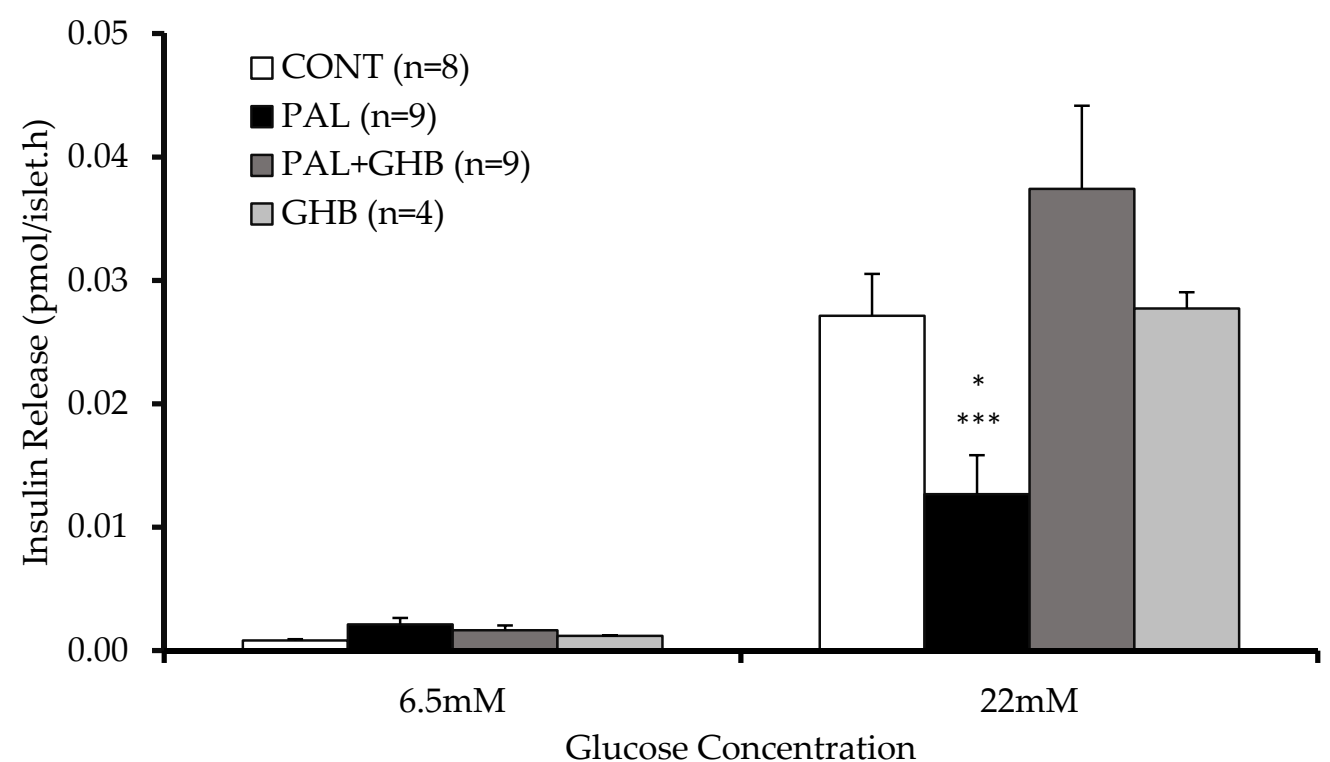

Figure 1. Effects of palmitate and gamma-hydroxybutyric acid (GHB) on glucose-stimulated insulin secretion in mouse islets. Islets were treated for $48 \mathrm{hrs}$ with (1) control media, (2) palmitate $(0.4 \mathrm{mM})$, (3) palmitate $(0.4 \mathrm{mM})$ and GHB (5 mM), and (4) GHB (5 mM) alone. GSIS was performed after $1 \mathrm{~h}$ recovery. Palmitate treatment impaired GSIS while GHB restored GSIS in palmitate treatment. GHB by itself did not affect GSIS. Data are means \pm SE. One-way nonparametric ANOVA on ranks for repeated measurements followed by Tukey's test was performed to compare differences between treatments. ${ }^{*} p<0.05$ vs. all, ${ }^{* * *} p<0.001$ vs. PAL + GHB.
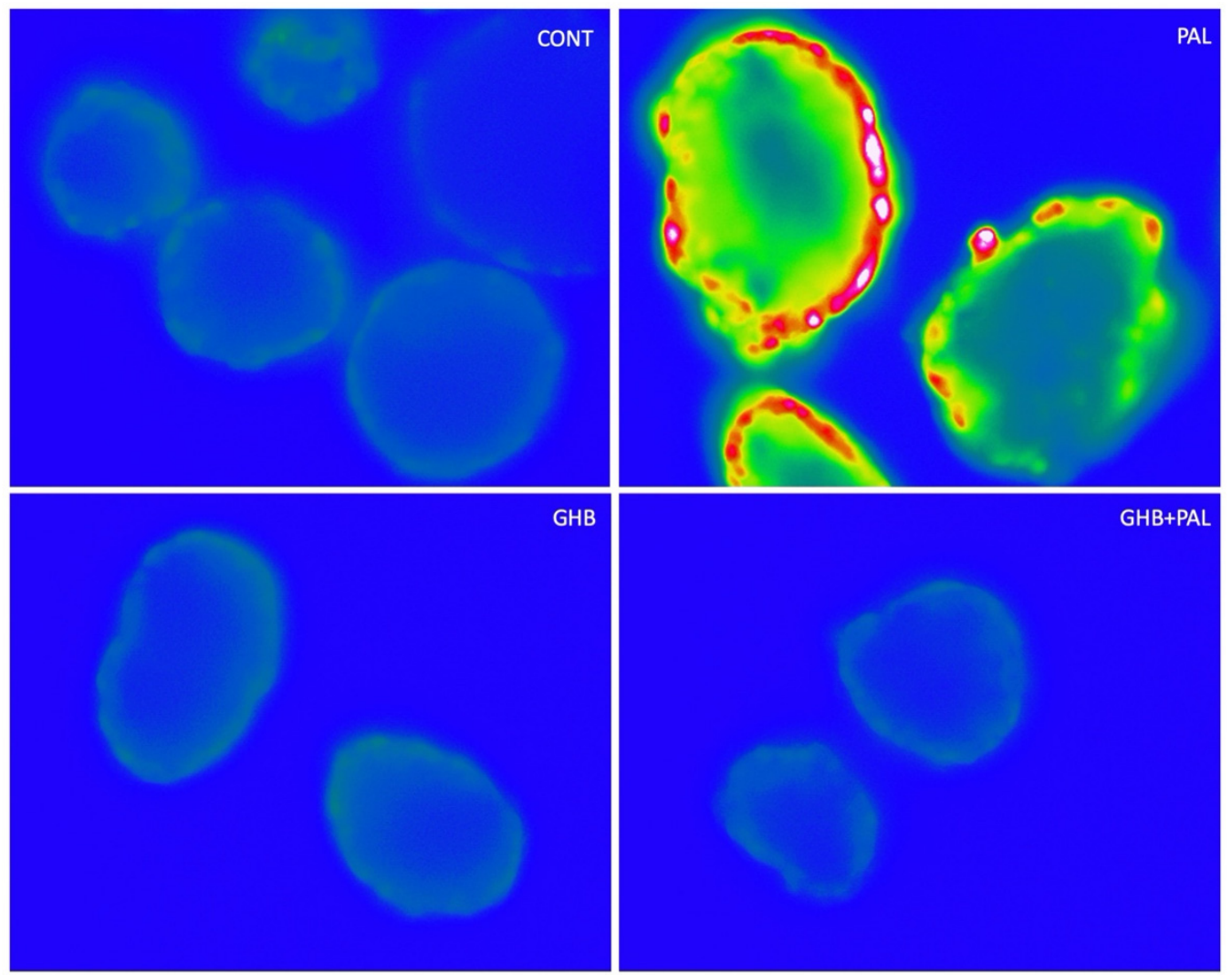

(a)

Figure 2. Cont. 


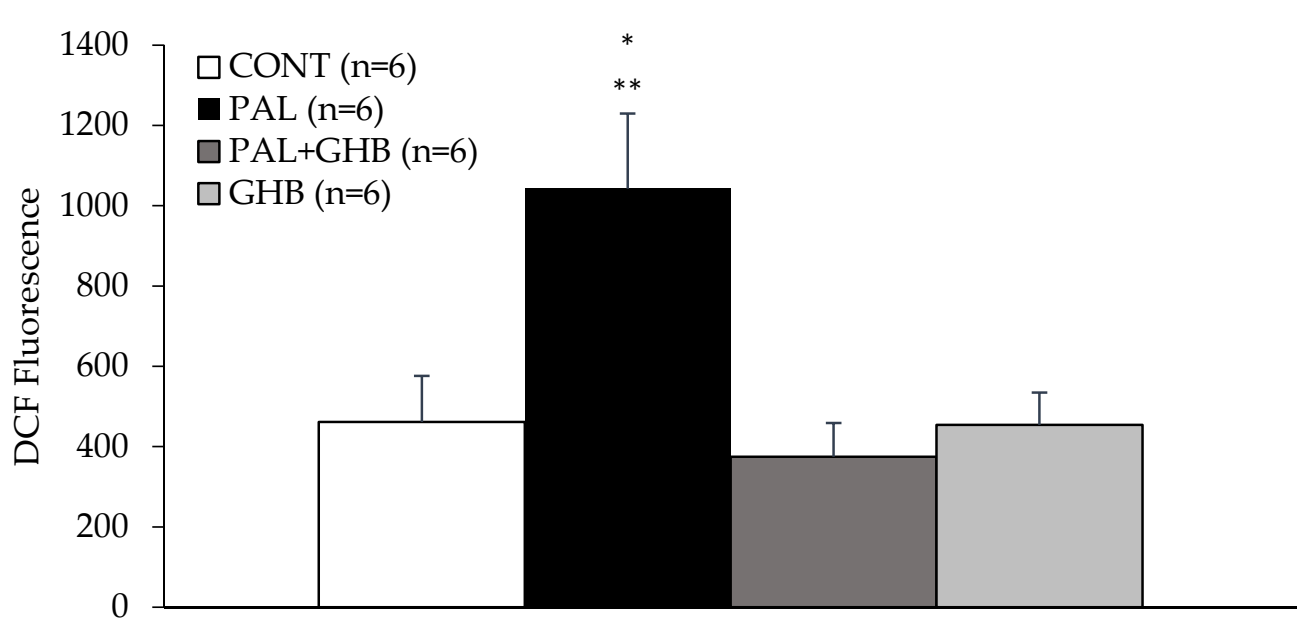

(b)

Figure 2. Effects of palmitate and gamma-hydroxybutyric acid (GHB) on ROS levels in mouse islets. ROS levels were determined by H2DCF-DA. Islets were treated for $48 \mathrm{hrs}$ with (1) control media, (2) palmitate $(0.4 \mathrm{mM})$, (3) palmitate $(0.4 \mathrm{mM})$ and GHB (5 mM), and (4) GHB (5 mM) alone. Approximately 10 islets were measured per experiment $(n)$. (a) Representative images. (b) Quantification of results. Palmitate treatment increased ROS while GHB had a protective effect. GHB alone did not affect H2DCF-DA-detected ROS. RFI = relative fluorescence intensity. Data are means \pm SE. One-way parametric ANOVA followed by Tukey's test was performed to compare differences between treatments. ${ }^{*} p<0.05$ vs. all, ${ }^{* *} p<0.01$ vs. PAL $+\mathrm{GHB}$.

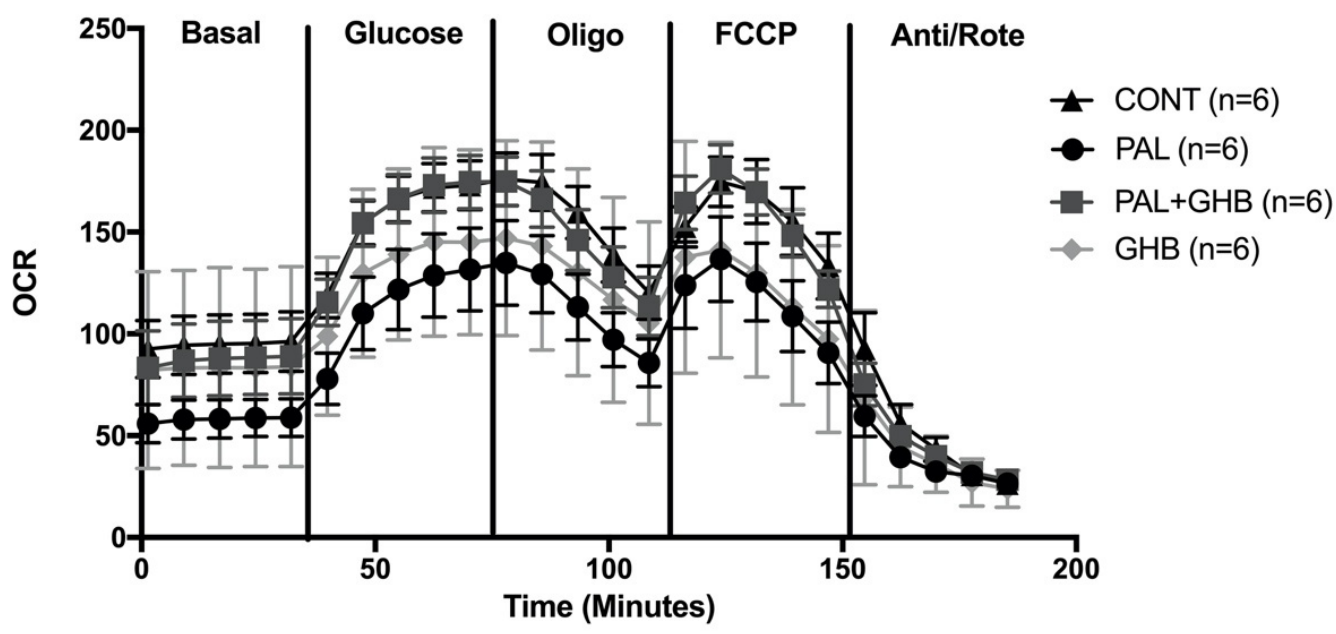

Figure 3. Effects of palmitate and gamma-hydroxybutyric acid (GHB) on oxygen consumption rate in mouse islets. Measurement of oxygen consumption rate (OCR) with Seahorse XF cell Mito Stress Test (Seahorse Bioscience, Billerica, MA, USA). Islets were treated for $48 \mathrm{hrs}$ with (1) control media (black triangle), (2) Palmitate (0.4 mM) (black circle), (3) Palmitate (0.4 mM) and GHB (5 mM) (dark-gray square), and (4) GHB (5 mM) alone (light-gray diamond). Palmitate treatment tended to reduce OCR while GHB seemed to have a protective effect. Data are means \pm SE. One-way parametric ANOVA for repeated measures followed by Tukey's test was performed to compare differences between treatments.

Figure 4a reveals that palmitate tended to reduce islet NADPH levels while cotreatment of palmitate with GHB significantly increased NADPH levels compared to islets treated only with palmitate $(p<0.01)$. Figure $4 \mathrm{~b}$ reveals that palmitate tended to increase islet NADP+ levels. Lastly, Figure 4c shows that palmitate reduced the ratio of 
$\mathrm{NADPH} / \mathrm{NADP}+$, which was prevented by co-treatment with GHB $(p<0.05$ vs. ALL, $p<0.01$ vs. PAL + GHB and GHB).

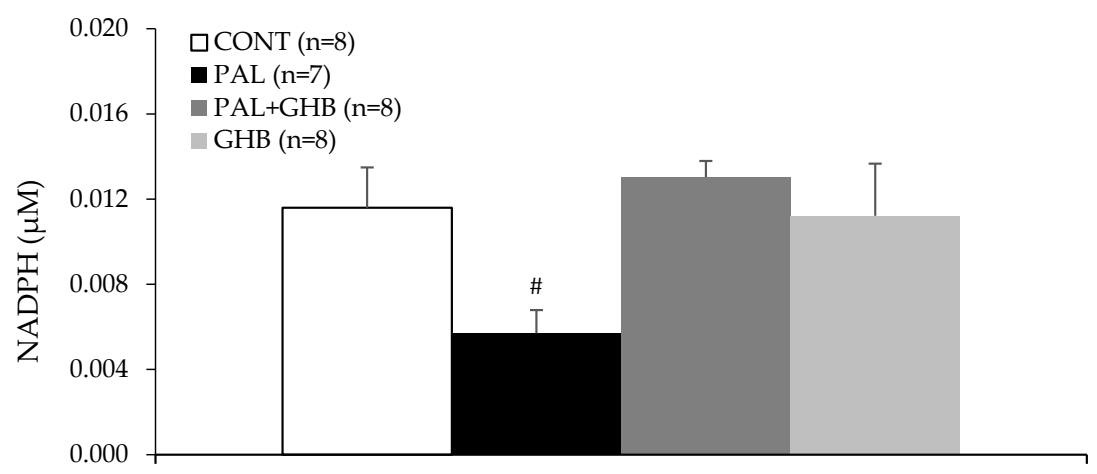

(a)

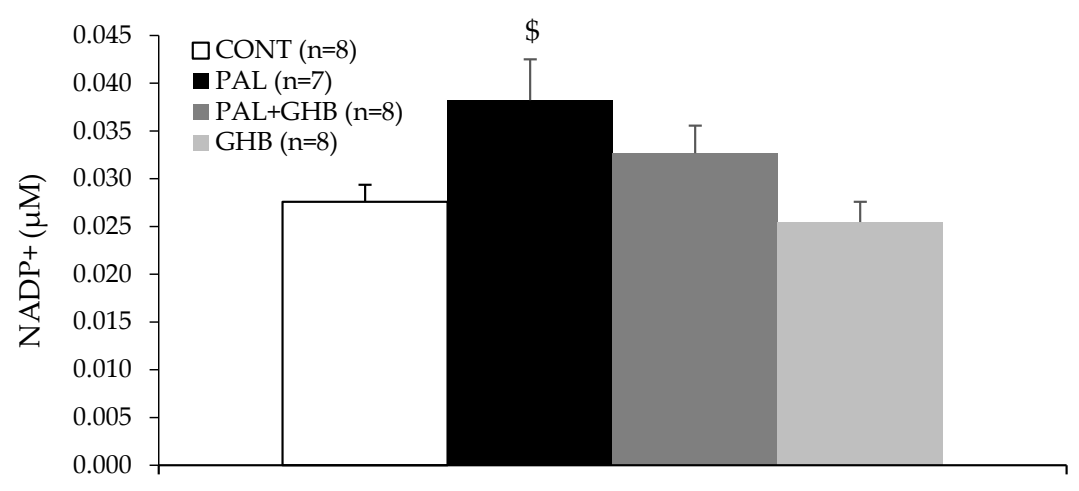

(b)

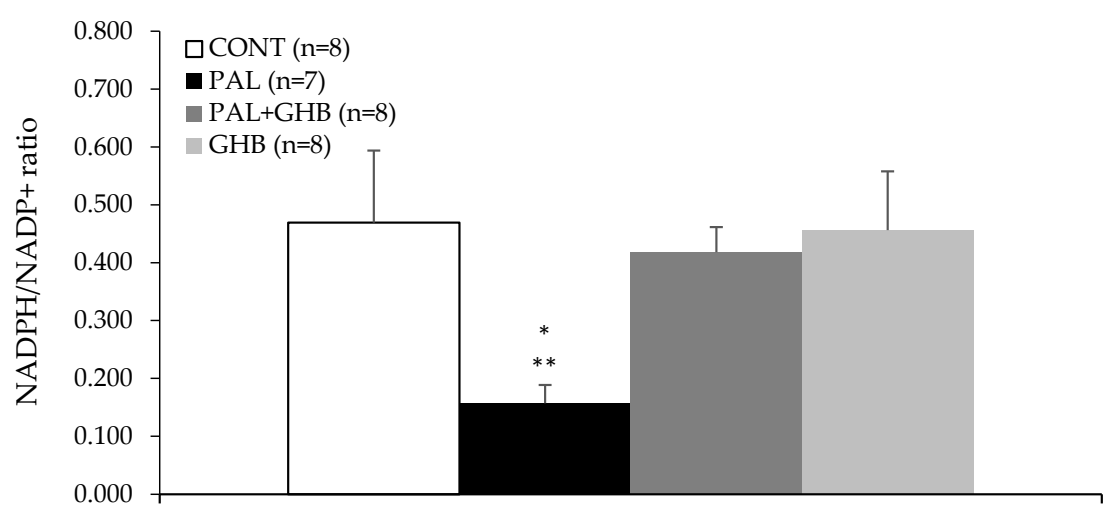

(c)

Figure 4. Effects of palmitate and gamma-hydroxybutyric acid (GHB) on NADPH and NADP+ in mouse islets. Islets were treated for $48 \mathrm{hrs}$ with (1) control media, (2) Palmitate (0.4 mM), (3) Palmitate $(0.4 \mathrm{mM})$ and GHB $(5 \mathrm{mM})$, and (4) GHB $(5 \mathrm{mM})$ alone. (a) NADPH measurement in islets. (b) NADP+ measurement in islets. (c) NADPH/NADP+ ratio in islets. Palmitate treatment reduced the NADPH/NADP+ ratio in islets while treatment with GHB reversed this effect. Palmitate tended to reduce NADPH levels and increase NADP+ in islets. Data are means $\pm \mathrm{SE}$. One-way parametric ANOVA followed by Tukey's test was performed to compare differences between treatments for NADP+. One-way nonparametric ANOVA on ranks followed by Tukey's test was performed to compare differences between treatments for NADPH and NADPH/NADP+ ratio. ${ }^{*} p<0.05 \mathrm{vs.} \mathrm{ALL;}$ ** $p<0.01$ vs. PAL + GHB and GHB; $\#<<0.01$ vs. PAL $+\mathrm{GHB} ; \$ p<0.05$ vs. GHB. 


\section{Discussion}

Exposure of pancreatic $\beta$-cells to NEFA induces the formation of ROS and impairs GSIS, a common measure of $\beta$-cell function [1,2]. Pancreatic $B$-cells have reduced antioxidant enzyme gene expression compared to other cells and correspondingly low levels of free radical detoxifying and redox regulating enzymes, which may account for their unusual vulnerability to ROS-induced oxidative stress [11]. Oxidative stress decreases insulin secretion by various mechanisms such as the induction of endoplasmic reticulum stress and inflammation, and the reduction of mitochondrial function and ultimately cell viability [13].

While ROS may be generated along the mitochondrial electron transport chain in the course of fatty acid oxidation or as a result of mitochondrial membrane damage, the major source of ROS generated in pancreatic $\beta$-cells in response to NEFA appears to be the formation of superoxide by the cytoplasmic/plasma membrane NADPH oxidase complex. NEFA have been shown to increase the activity of NADPH oxidase in islets [14,15], in part by PKC activation [16].

Our pharmacological and genetic studies confirm the role of NADPH oxidase in the generation of ROS by NEFA and the role of these ROS in the impairment of $B$-cell function in vivo [2]. NADPH is a substrate for NADPH oxidase, and its oxidation should reduce the NADPH/NADP+ ratio in the cell, as found in the present study. However, measurements indicate that NEFA may not affect or actually increase the intracellular $\mathrm{NADPH} / \mathrm{NADP}+$ ratio $[2,17,18]$. In addition to the PPS, there are two other major sources of NADPH. A mitochondrial enzyme, nicotinamide nucleotide transhydrogenase, can form NADPH from NADH; however, this enzyme is absent in this strain of C57BL/ 6 mice from Jackson [19]. NADPH can also be formed from cytosolic malate as described below when acetyl-CoA in mitochondria produced in the course of NEFA oxidation allosterically activates pyruvate carboxylase to form oxaloacetate. This important anaplerotic reaction is followed by the conversion of oxaloacetate to malate by malate dehydrogenase. Malate then enters the cytoplasm, where it is decarboxylated to pyruvate by the malic enzyme together with the formation of NADPH. This malate-pyruvate shunt can produce far more NADPH than the PPS, which is known to be a very minor route of glucose metabolism in pancreatic $\beta$-cells. Glucose and succinate, which are both precursors of pyruvate and malate, can also activate the shunt and generate NADPH. However, the prolonged exposure of $\beta$-cells to NEFA suppresses pyruvate carboxylase gene transcription and the NADPH-generating activities of the malate pyruvate shunt [20], which may further explain the decrease in the $\mathrm{NADPH} / \mathrm{NADP}+$ ratio seen with palmitate in the present study.

One important function of NADPH in the $B$-cell is to serve as a cofactor for antioxidant enzymes, including glutathione reductase, thiol reductase, and quinone reductase. Decreased formation of NADPH thus elevates the level of ROS. The rise in the level of ROS may then uncouple the electron transport chain, interfere with the synthesis of ATP and insulin secretion, and ultimately threaten the viability of the $B$-cells [18].

The NADPH/NADP+ ratio also regulates the response of the $\mathrm{B}$-cells to two major insulin secretagogues, glucose and succinate. Both of these secretagogues enhance the formation of NADPH. The flow of electrons from NADPH to glutathione and to the redox proteins glutaredoxin and thioredoxin mediates insulin granule exocytosis [21]. Insulin release is also regulated by the NADPH/NADP+ ratio's effect on Kv channels. Active $\mathrm{Kv}$ channels repolarize the B-cells. A high intracellular NADPH/NADP+ ratio inactivates these $\mathrm{Kv}$ channels and thus increases insulin secretion by maintaining membrane depolarization [22].

Our findings indicate that GHB is effective at blocking the formation of ROS and preventing the inhibitory effect of the NEFA palmitate on GSIS. GHB's antioxidant powers have been attributed to its capacity to activate the PPS and generate NADPH $[6-8,23]$. In mice, in vivo, high intraperitoneal doses of GHB $(500 \mathrm{mg} / \mathrm{kg})$ increase the ratio of $1-14 \mathrm{C} / 6$ $14 \mathrm{C}$ in expired air, a measure of the shift in glucose metabolism to the PPS, by $300 \%$. The same effect is seen with slices of cerebral cortical grey matter in rats but not with slices of kidney or diaphragm. Intraperitoneal doses of $500 \mathrm{mg} / \mathrm{kg}$ in mice and rats increase 
glucose-6-phosphate dehydrogenase activity in the whole brain by $27 \%$. However, the activity of glucose-6-phosphate dehydrogenase in vitro is not altered by high concentrations of GHB [8]. Taberner et al. [8] were not able to explain how GHB activated the PPS and generated NADPH, and, to our knowledge, the reason for this activation has never been satisfactorily explained. Glucose-6-phosphate dehydrogenase, a cytosolic enzyme, is the rate-limiting step in the PPS and is not known to be activated by GHB and, in fact, is negatively regulated by an increasing NADPH/NADP+ ratio. Utilization of NADPH and the ensuing rise in the level of NADP+ activates the enzyme [24].

GHB is metabolized in both the cytoplasm and mitochondria [25,26]. An NADP+ dependent oxidoreductase oxidizes GHB in the cytoplasm to succinic semialdehyde with the concomitant formation of NADPH. This reaction is inhibited by NADPH and proceeds slowly but it is greatly accelerated in the brain, liver, and kidney when it is coupled to the reduction of D-glucuronate to L-gulonate by NADPH and the regeneration of NADP+. The coupled reaction results in the rapid formation of succinic semialdehyde without any overall change in the levels of either NADPH or NADP+ $[25,26]$. However, this has not been investigated in the $ß$-cell. In mitochondria, a hydroxyacid-oxoacid transhydrogenase catalyzes the transformation of GHB and $\alpha$-ketoglutarate to succinic semialdehyde and $\mathrm{D}$ - $\alpha$-hydroxyglutarate. In both cases, succinic semialdehyde is rapidly converted to succinic acid with the concomitant reduction of NAD+ to NADH $[25,26]$.

In our in vitro experimental set-up, islets were incubated for $48 \mathrm{~h}$ in the presence of palmitate with or without GHB. ROS formation was measured after the end of this incubation period. During these $48 \mathrm{~h}$, the catabolism of GHB and the formation of high levels of succinate may have activated the malate-pyruvate shunt impaired by palmitate and generated the antioxidant cofactor NADPH. Alternatively, GHB may have activated the PPS. The high levels of NADH generated by the oxidation of succinic semialdehyde may activate nicotinamide nucleotide transhydrogenase to form additional NADPH [27], however as stated previously, this enzyme is lacking in C57BL/6J mice. Once generated, NADPH may have affected insulin secretion by its antioxidant mechanism as well as by acting on exocytosis and $\mathrm{Kv}$ channels, as described earlier.

Although the catabolism of GHB has been shown to generate NADH and succinate in the brain and in peripheral tissues, past studies have demonstrated that GHB only increases oxygen consumption in slices of cerebral grey matter $[8,26]$. GHB was not able to increase oxygen consumption in cerebral homogenates or in liver slices and, in our study, it was not able to increase oxygen consumption in isolated islets. Oxygen consumption actually tended to be depressed by GHB alone, which could be explained by the diversion of $\alpha$-ketoglutarate to $D$ - $\alpha$-hydroxyglutarate in the Krebs cycle. The generation of ROS in response to palmitate may account for the reduction in oxygen consumption via mitochondrial dysfunction, and GHB appeared to prevent this reduction presumably via its antioxidant effect. The effect of GHB to improve mitochondrial function in the presence of palmitate was, however, not significant and was minor compared to its effect on palmitateinduced ROS, which suggests that other mechanisms related to GHB's antioxidant effect or to the NADPH elevation contributed to the GHB effect on GSIS.

GHB has been shown to have widespread cellular protective effects $[3,4]$. Protection against ischemic reperfusion injury has been demonstrated in the brain, gut, liver, and vascular endothelium. Thus, in addition to protecting the $B$-cells of the pancreas from the toxic effects of high levels of NEFA in diabetes, GHB may also be able to protect the vascular endothelium in diabetes from the high levels of oxidative stress and vascular damage that leads in time to major end organ failure [28,29]. Long-acting formulations of GHB are now being developed for once nightly use (Avadel Pharmaceuticals, Dublin, Ireland; XW Pharma Inc., Redwood City, CA, USA) This may facilitate its potential use as an adjuvant in patients with diabetes and sleep disorders. Stress and poor sleep have long been recognized to interfere with the optimum control of the disease. Insufficient sleep is recognized to alter the regulation of energy intake and circadian timing and to lead to weight gain, insulin resistance, and glucose intolerance [30]. It might also be possible to prevent the central 
effects of GHB by PEGylating the molecule, which impedes the blood-brain barrier passage, as recently shown with other compounds [31,32], while retaining its favorable peripheral antidiabetic effects.

\section{Conclusions}

GHB is shown to be effective at quenching the generation of ROS in response to the NEFA palmitate, and in preventing the impairment of GSIS normally caused by palmitate. GHB's protective actions may be related to its capacity to stimulate the formation of the antioxidant cofactor NADPH.

Author Contributions: Conceptualization: A.G. and M.M.; methodology: A.G.; formal analysis: J.H.M.Y. and L.S.N.Y.; investigation and data curation: J.H.M.Y., L.S.N.Y., A.I., Y.F.T., B.B., H.G. and E.M.J.; resource: A.G., M.B.W. and J.W.J.; writing—original draft preparation: J.H.M.Y. and M.M.; writing—review and editing,: A.G. and M.M.; visualization and supervision: M.B.W., J.W.J. and A.G.; project administration and funding acquisition: A.G. All authors have read and agreed to the published version of the manuscript.

Funding: This research was funded by Jazz Pharmaceuticals grant number 2014-0285.

Institutional Review Board Statement: The animal study protocol was approved by the Animal Care Committee of the University of Toronto (animal protocol number: 20011526, approved 30 March 2021).

Informed Consent Statement: Not applicable.

Data Availability Statement: The data presented in this study are available on request to the corresponding author.

Acknowledgments: The authors thank Loretta Lam for excellent technical assistance.

Conflicts of Interest: The authors declare no conflict of interest. In particular, the authors have no shares in Jazz Pharmaceuticals. The authors thank Jazz Pharmaceuticals for funding of this IST research grant. Jazz Pharmaceuticals also reviewed the manuscript and had the opportunity to provide suggestions to the authors for their consideration. Although Jazz Pharmaceuticals reviewed the content of this manuscript, the ultimate interpretation, and the decision to submit it for publication was made by the authors independently. Dr. Mamelak is a consultant to XW Pharma Inc.

\section{References}

1. Oprescu, A.I; Bikopoulos, G.; Naassan, A.; Allister, E.M.; Tang, C.; Park, E.; Uchino, H.; Lewis, G.F.; Fantus, I.G.; Rozakis-Adcock, M.; et al. Free Fatty Acid-Induced Reduction in Glucose-Stimulated Insulin Secretion: Evidence for a Role of Oxidative Stress In Vitro and In Vivo. Diabetes 2007, 56, 2927-2937. [CrossRef] [PubMed]

2. Koulajian, K.; Desai, T.; Liu, G.C.; Ivovic, A.; Patterson, J.N.; Tang, C.; El-Benna, J.; Joseph, J.W.; Scholey, J.W.; Giacca, A. NADPH oxidase inhibition prevents beta cell dysfunction induced by prolonged elevation of oleate in rodents. Diabetologia 2013, 56, 1078-1087. [CrossRef] [PubMed]

3. Mamelak, M.; Hyndman, D. Gammahydroxybutyrate and oxidative stress. In Gammahydroxybutyrate: Molecular, Functional and Clinical Aspects; Tunnicliff, G., Cash, C., Eds.; Taylor and Francis: New York, NY, USA, 2002; pp. 218-235.

4. Mamelak, M. Energy and the Alzheimer brain. Neurosci. Biobehav. Rev. 2017, 75, 297-313. [CrossRef] [PubMed]

5. Bouix, O.; Reynier, M.; Guintrand-Hugret, R.; Orsetti, A. Protective effect of gamma-hydroxybutyrate and nicotinamide on low-dose streptozotocin-induced diabetes in mice. Horm. Metab. Res. 1995, 27, 216-220. [CrossRef] [PubMed]

6. Pierrefiche, G.; Topall, G.; Henriet, I.; Laborit, H. Protective effect of gamma-hydroxybutyrate on alloxan induced diabetes in mice. Res. Commun. Chem. Pathol. Pharmacol. 1991, 71, 309-319.

7. Laborit, H. Sodium 4-hydroxybutyrate. Int. J. Neuropharmacol. 1964, 3, 433-IN8. [CrossRef]

8. Taberner, P.V.; Rick, J.T.; Kerkut, G.A. The Action of Gamma-Hydroxybutyric Acid on Cerebral Glucose Metabolism. J. Neurochem. 1972, 19, 245-254. [CrossRef]

9. Abad, V.C. An evaluation of sodium oxybate as a treatment option for narcolepsy. Expert Opin Pharm. 2019, 20, 1189-1199. [CrossRef]

10. Joseph, J.W.; Koshkin, V.; Saleh, M.C.; Sivitz, W.I.; Zhang, C.-Y.; Lowell, B.B.; Chan, C.B.; Wheeler, M.B. Free Fatty Acid-induced B-Cell Defects Are Dependent on Uncoupling Protein 2 Expression. J. Biol. Chem. 2004, 279, 51049-51056. [CrossRef]

11. Lenzen, S.; Drinkgern, J.; Tiedge, M. Low antioxidant enzyme gene expression in pancreatic islets compared with various other mouse tissues. Free. Radic. Biol. Med. 1996, 20, 463-466. [CrossRef] 
12. Ivovic, A.; Oprescu, A.I.; Koulajian, K.; Mori, Y.; Eversley, J.A.; Zhang, L.; Nino-Fong, R.; Lewis, G.F.; Donath, M.Y.; Karin, M.; et al. IKKB inhibition prevents fat-induced beta cell dysfunction in vitro and in vivo in rodents. Diabetologia 2017, 60, $2021-2032$. [CrossRef]

13. Giacca, A.; Xiao, C.; Oprescu, A.I.; Carpentier, A.C.; Lewis, G.F. Lipid-induced pancreatic ß-cell dysfunction: Focus on in vivo studies. Am. J. Physiol. -Endocrinol. Metab. 2011, 300, E255-E262. [CrossRef]

14. Morgan, D.; Oliveira-Emilio, H.R.; Keane, D.; Hirata, A.E.; Da Rocha, M.S.; Bordin, S.; Curi, R.; Newsholme, P.; Carpinelli, A.R. Glucose, palmitate and pro-inflammatory cytokines modulate production and activity of a phagocyte-like NADPH oxidase in rat pancreatic islets and a clonal beta cell line. Diabetologia 2007, 50, 359-369. [CrossRef]

15. Newsholme, P.; Haber, E.P.; Hirabara, S.M.; Rebelato, E.L.O.; Procopio, J.; Morgan, D.; Oliveira-Emilio, H.C.; Carpinelli, A.R.; Curi, R. Diabetes associated cell stress and dysfunction: Role of mitochondrial and non-mitochondrial ROS production and activity. J Physiol. 2007, 583, 9-24. [CrossRef]

16. Fontayne, A.; Dang, P.M.-C.; Gougerot-Pocidalo, A.M.-A.; El Benna, J. Phosphorylation of p47phox sites by PKC alpha, beta II, delta, and zeta: Effect on binding to p22phox and on NADPH oxidase activation. Biochemistry 2002, 41, 7743-7750. [CrossRef]

17. Macdonald, M.J. Feasibility of a Mitochondrial Pyruvate Malate Shuttle in Pancreatic Islets Further Implication of Cytosolic Nadph in Insulin Secretion. J. Biol. Chem. 1995, 270, 20051-20058. [CrossRef]

18. Pike, L.S.; Smift, A.L.; Croteau, N.J.; Ferrick, D.A.; Wu, M. Inhibition of fatty acid oxidation by etomoxir impairs NADPH production and increases reactive oxygen species resulting in ATP depletion and cell death in human glioblastoma cells. Biochim. Biophys. Acta (BBA)-Bioenerg. 2011, 1807, 726-734. [CrossRef]

19. Ronchi, J.A.; Figueira, T.R.; Ravagnani, F.G.; Oliveira, H.C.F.; Vercesi, A.E.; Castilho, R.F. A spontaneous mutation in the nicotinamide nucleotide transhydrogenase gene of C57BL/6J mice results in mitochondrial redox abnormalities. Free. Radic. Biol. Med. 2013, 63, 446-456. [CrossRef]

20. Iizuka, K.; Nakajima, H.; Namba, M.; Miyagawa, J.-I.; Miyazaki, J.-I.; Hanafusa, T.; Matsuzawa, Y. Metabolic consequence of long-term exposure of pancreatic $B$ cells to free fatty acid with special reference to glucose insensitivity. Biochim. Biophys. Acta (BBA)-Mol. Basis Dis. 2002, 1586, 23-31. [CrossRef]

21. Ivarsson, R.; Quintens, R.; Dejonghe, S.; Tsukamoto, K.; Veld, P.I.; Renström, E.; Schuit, F.C. Redox Control of Exocytosis: Regulatory Role of NADPH, Thioredoxin, and Glutaredoxin. Diabetes 2005, 54, 2132-2142. [CrossRef]

22. MacDonald, P.E.; Wheeler, M.B. Voltage-dependent $\mathrm{K}+$ channels in pancreatic beta cells: Role, regulation and potential as therapeutic targets. Diabetologia 2003, 46, 1046-1062. [CrossRef] [PubMed]

23. Lopatin, A.F.; Riabtseva, E.G.; Riabova, V.V.; TIu, L. Effect of sodium oxybate on metabolic indices in ischemis hypoxia of muscle tissue. Farmakol. Toksikol. 1984, 47, 53-55. [PubMed]

24. Stanton, R.C. Glucose-6-Phosphate Dehydrogenase, NADPH, and Cell Survival. IUBMB Life 2012, 64, 362-369. [CrossRef] [PubMed]

25. Kaufman, E.E.; Nelson, T. An overview of gamma-hydroxybutyrate catabolism: The role of the cytosolic NADP(+)-dependent oxidoreductase EC 1.1.1.19 and of a mitochondrial hydroxyacid-oxoacid transhydrogenase in the initial, rate-limiting step in this pathway. Neurochem. Res. 1991, 16, 965-974. [CrossRef] [PubMed]

26. Kaufman, E.E. Metabolism and distribution of gammahydroxybutyrate in the brain. In Gammahydroxybutyrate: Molecular, Functional and Clinical Aspects; Hyndman, D., Cash, C., Eds.; Taylor and Francis: New York, NY, USA, 2002; pp. 1-16.

27. Vogel, R.; Wiesinger, H.; Hamprecht, B.; Dringen, R. The regeneration of reduced glutathione in rat forebrain mitochondria identifies metabolic pathways providing the NADPH required. Neurosci. Lett. 1999, 275, 97-100. [CrossRef]

28. Förstermann, U.; Xia, N.; Li, H. Roles of Vascular Oxidative Stress and Nitric Oxide in the Pathogenesis of Atherosclerosis. Circ. Res. 2017, 120, 713-735. [CrossRef] [PubMed]

29. Sena, C.M.; Pereira, A.M.; Seiça, R. Endothelial dysfunction-A major mediator of diabetic vascular disease. Biochim. Biophys. Acta (BBA)-Mol. Basis Dis. 2013, 1832, 2216-2231. [CrossRef] [PubMed]

30. Depner, C.M.; Stothard, E.R.; Wright, K.P. Metabolic consequences of sleep and circadian disorders. Curr. Diabetes Rep. 2014, 14, 507. [CrossRef]

31. Garnock-Jones, K.P. Naloxegol: A Review of Its Use in Patients with Opioid-Induced Constipation. Drugs 2015, 75, 419-425. [CrossRef]

32. Mahú, I.; Barateiro, A.; Rial-Pensado, E.; Martinez-Sanchez, N.; Vaz, S.; Cal, P.M.; Jenkins, B.; Rodrigues, T.; Cordeiro, C.; Costa, M.F.; et al. Domingos, Brain-Sparing Sympathofacilitators Mitigate Obesity without Adverse Cardiovascular Effects. Cell Metab. 2020, 31, 1120-1135.e7. [CrossRef] 